\title{
PAROSTEAL OSTEOGENIC SARCOMA OF BONE
}

\author{
Report of a Case \\ W. J. Weston, J. D. Reid and J. H. Saunders, Wellington, New Zealand
}

From the Wellington and Hutt Hospitals, New Zealand

In 1949 and in 1951 Geschickter and Copeland described as "parosteal osteoma " a tumour occurring in adults, usually at the back of the lower end of the femur and bulging into the popliteal fossa. This was considered to start as a benign proliferation of ossifying fibrous tissue arising in the region of the periosteum, resembling myositis ossificans in some respects but involving neighbouring bone. Over a number of years this tended to evolve into a malignant tumour histologically akin to sclerosing osteogenic sarcoma. In 1954 Dwinnell, Dahlin and Ghormley reviewed fifteen cases under the title of "parosteal (juxtacortical) osteogenic sarcoma," concluding that the tumour was of low-grade malignancy from the start. They found no good evidence that myositis ossificans was implicated, and no evidence of any other pre-existing benign lesion. In spite of the difficulties in establishing histological evidence of malignancy they felt that the clinical behaviour of the tumour justified early amputation.

Lichtenstein (1955), however, regarded these lesions as juxta-cortical tumour-like masses of metaplastic bone or cartilage or both, in which malignant change was uncommon and for which amputation should not be done without convincing pathological proof of malignancy. Although the lesion was analogous to myositis ossificans elsewhere, he conceded a greater liability to malignant change when the situation was parsoteal, with slow and subtle development of one type of " periosteal," " parosteal" or " juxta-cortical" osteogenic sarcoma. In this he followed Jaffe and Selin (1951) who, in describing the more favourable course of their juxta-cortical type of osteogenic sarcoma, thought that this tumour might represent the parosteal counterpart of the malignant form of myositis ossificans.

Fine and Stout (1956) stated that parosteal osteoma, unlike the benign implication of its name, was a malignant tumour, capable of metastasising, and distinguished from soft-tissue osteogenic sarcoma only by its slow progression from histologically benign to malignant tumour and by its attachment to, and involvement of, bone.

The following case is reported because of histological evidence suggesting a benign tumour with sarcomatous change. There was also radiological evidence of malignancy, and abnormal arteries of a type not described in the literature were seen. The histological nature of these is described.

\section{CASE REPORT}

A married woman aged thirty-six years developed an ache behind her left knee. Six months later a lump was noticed, but operation was refused because of an impending trip to England. In London, in July 1954, about seven months after the lump had been found, biopsy was performed by $\mathrm{Mr} \mathrm{H}$. W. Wright. The histological examination (by $\mathrm{Dr} \mathrm{L}$. Woodhouse Price) showed dense fibrous tissue admixed with delicate collagen fibrils and fibroblastic cells (Fig. 1). Dr Price considered that the lesion was essentially fibromatous, and that if it were a fibrosarcoma the degree of malignancy was manifestly extremely low. The patient was first seen in the Hutt Hospital in April 1955, when the ache behind the knee was increasing in severity, especially at night, and was aggravated by heat. She had no other relevant complaints, and the family and past history were irrelevant. On examination a fixed smooth bony mass was palpable in the upper part of the left popliteal fossa, about three inches long and two 
inches wide. It extended round the lateral aspect of the femur and was slightly tender proximally. There was no surrounding induration and only minor restriction of knee flexion. There were no palpable inguinal glands and there was no muscle wasting. The laboratory examinations were normal except that the erythrocyte sedimentation rate was 28 millimetres in the first hour.

Plain radiographs showed a mass of new bone on the cortex of the posterior and lateral aspects of the lower end of the femur. Posteriorly, transradiant areas through the new bone suggested islands of cartilage. In many parts the surface of the new bone was smooth and well defined, but, behind, irregular strands extended into the soft tissues perpendicularly to the shaft. Anteriorly there was some rarefaction of the cortex and the underlying bone. The lung fields were clear.

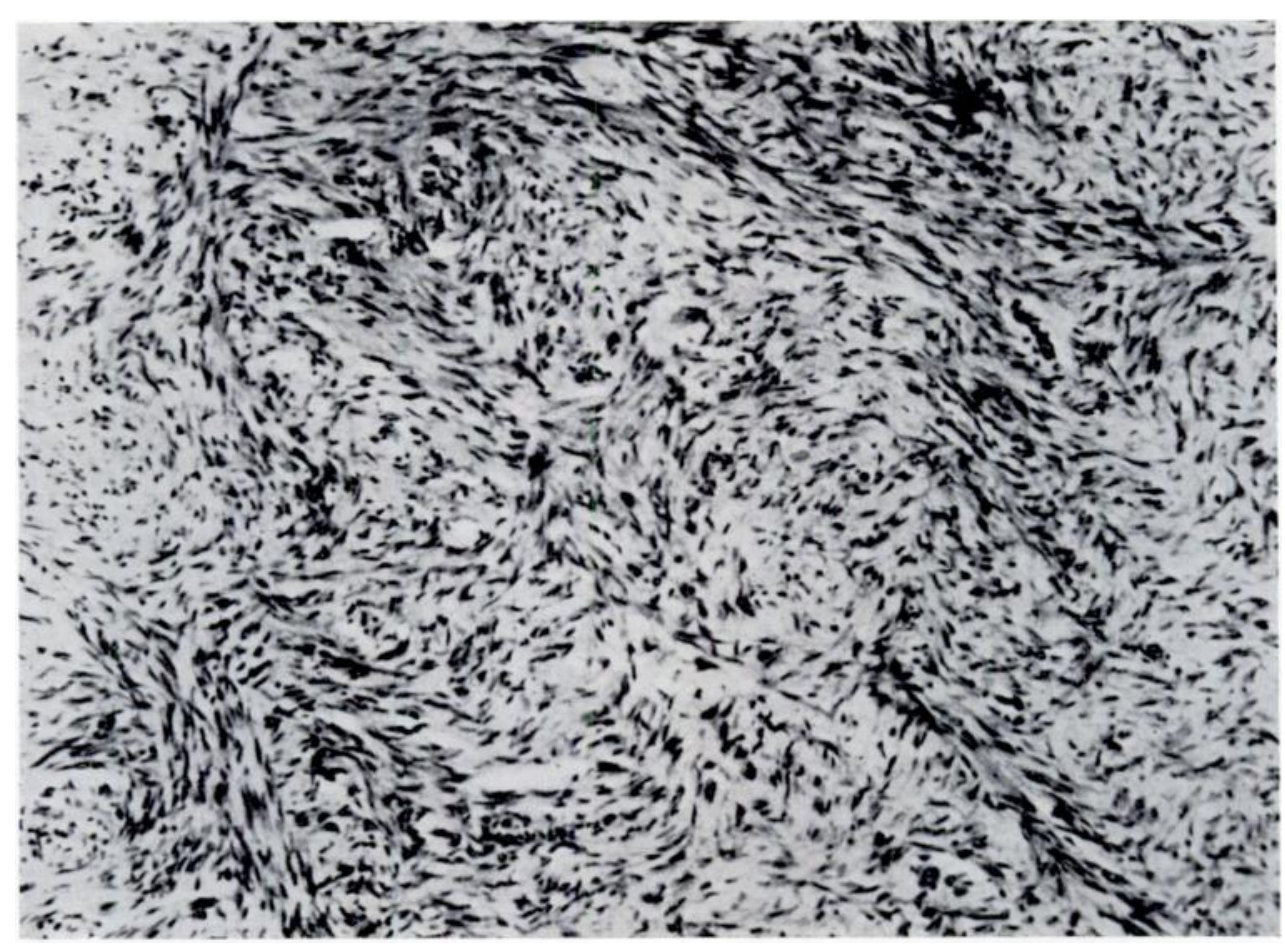

Fig. 1

Section shows dense fibrous tissue admixed with delicate fibrils and fibroblastic cells. Original biopsy in London 1954.

A left femoral arteriograph showed an increased regional circulation with a vascular plexus in and on the tumour at three main points (Figs. 2 and 3). Several arteriovenous shunts could be seen in the arterial phase. There were two pathological arteries with localised constrictions and dilations along their courses, presenting the "bow-tie" appearance (Fig. 4). Final radiological diagnosis was osteochondrosarcoma.

Biopsy specimens were taken at three widely separated points. The first specimen consisted of several portions of white fibrous tissue with attached bony fragments. Sections showed irregular trabeculae of bone with intervening fibrous tissue containing very occasional giant cells. There was no evidence of sarcomatous change. Another specimen consisted of a piece of firm white fibrous tissue measuring $2.5 \times 1 \times 0.5$ centimetres, one end of which contained a few fragments of bone. The sections showed a mass of dense cellular fibrous tissue with trabeculae of bone. There was one small island of cartilage. The third specimen consisted of a flattened piece of bony tissue measuring $2.5 \times 2 \times 0.5$ centimetres. The sections 


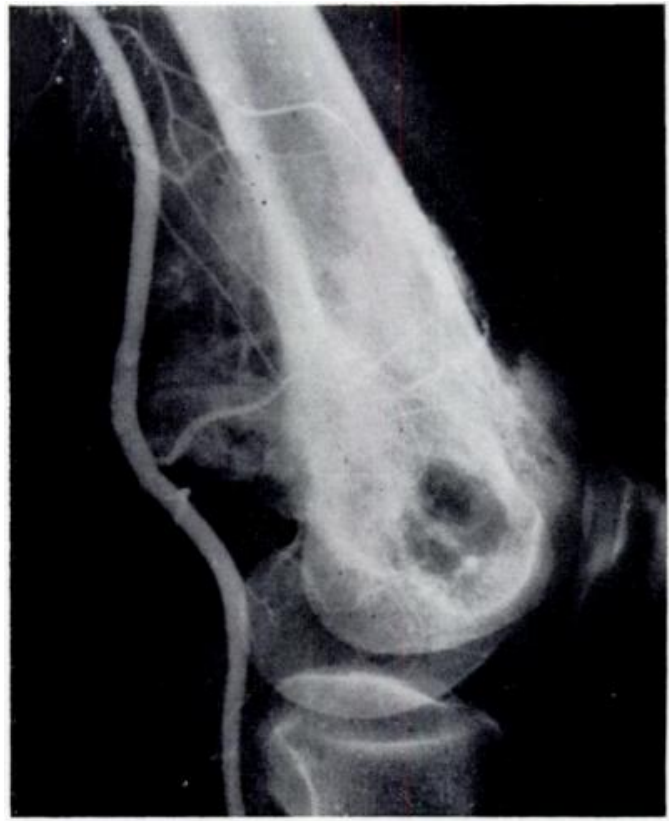

Fig. 2

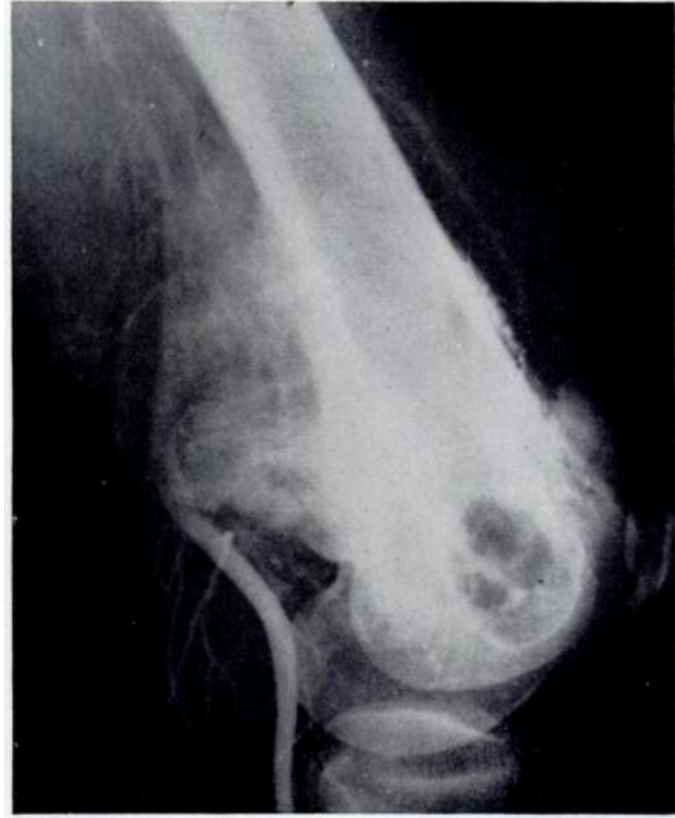

Fig. 3

Serial arteriographs showing venous filling in the arterial phase at three main points. They are taken at three seconds (Fig. 2) and five seconds (Fig. 3) after the injection of the radio-opaque fluid into the femoral artery. There is increased regional circulation and pathological arteries are outlined.

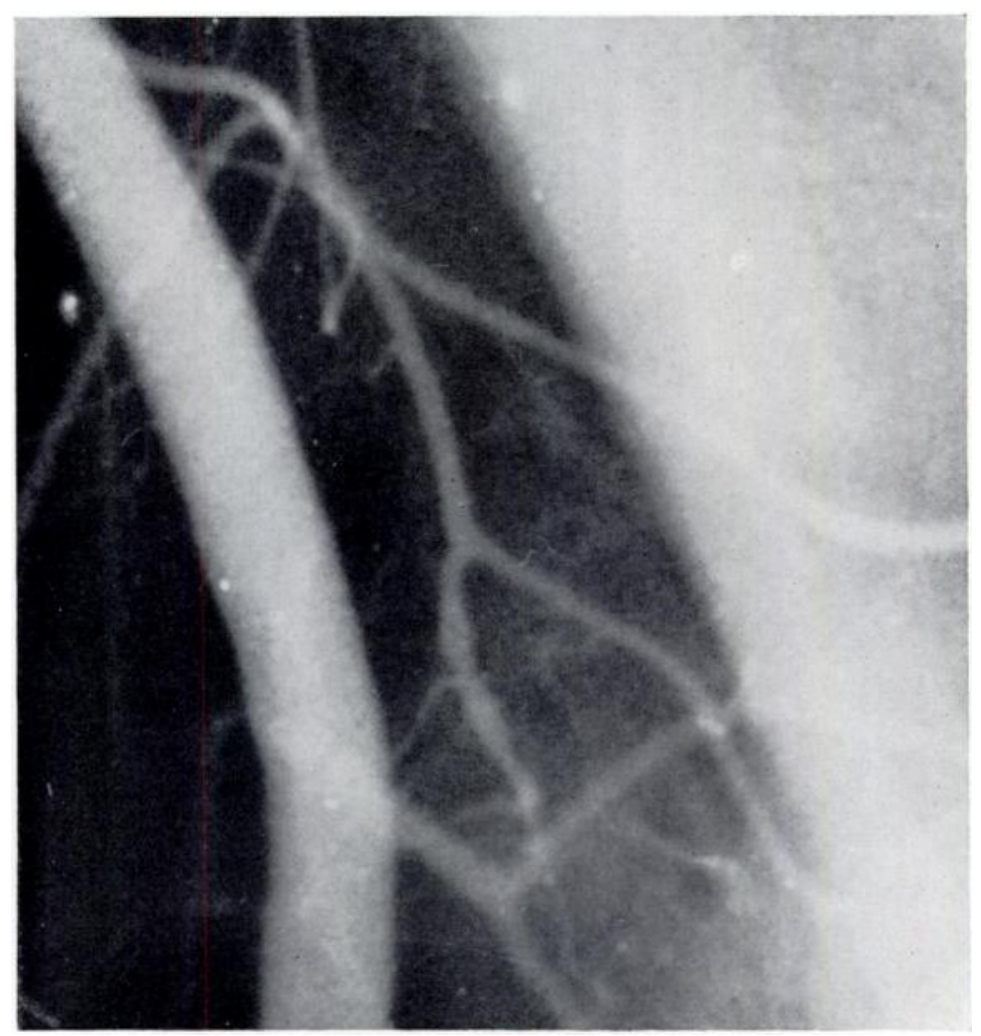

FIG. 4

Enlarged arteriograph showing the " bow-tie" appearance in the pathological arteries. 
showed fibrous tissue involving striated muscle with bone formation in the fibrous areas. The process had involved the adjacent fatty tissue. The sections indicated that this was a fibrous tumour extending into muscle and undergoing ossification. There was no evidence of malignancy. The conclusion was that the tumour was a parosteal osteoma.

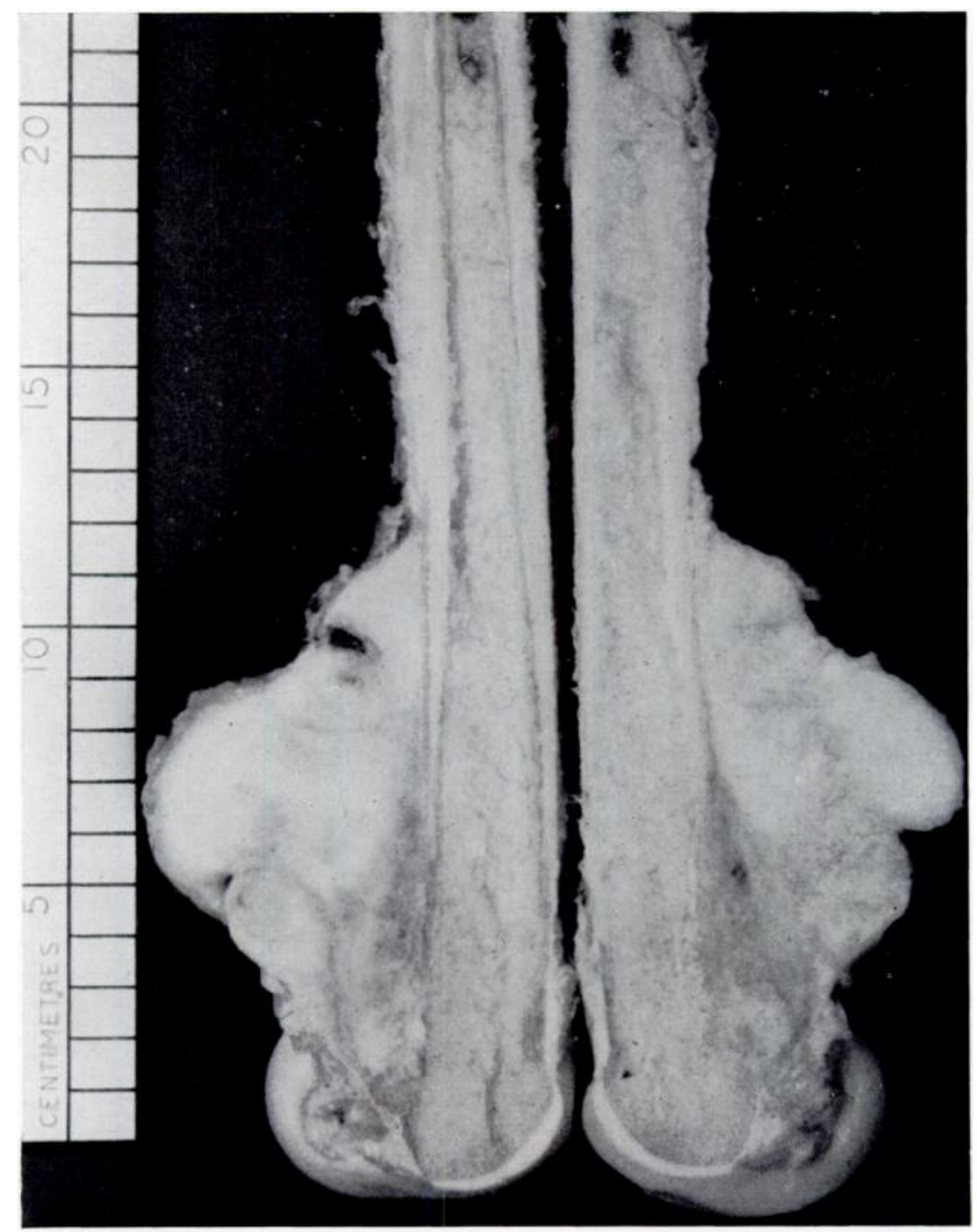

Fig. 5

Midline sagittal section of the tumour at the lower end of the femur. The posterior cortical bone is destroyed.

The left leg was amputated two months later through the mid-thigh leaving an eight-inch stump. Sixteen months later the patient was well and there was no evidence of recurrence. The latest follow-up (from Australia) in March 1958 stated that the patient was fit and was eight weeks pregnant.

Examination of the specimen-To enable histological examination of the abnormal arteries demonstrated radiographically, coloured gelatin was injected into the femoral artery, and vessels were dissected out and identified by comparison with radiographs.

Dissections revealed a bony mass on the posterior surface of the lower part of the femur, extending along it proximally for 9.5 centimetres, and almost enveloping the shaft from either side. Proximally there was obvious extension into muscle. On midline sagittal section the growth was predominantly extra-periosteal (Fig. 5). The posterior cortex was intact proximally

VOl. $40 \mathrm{~B}$, NO. 4, NOVEMBER 1958 


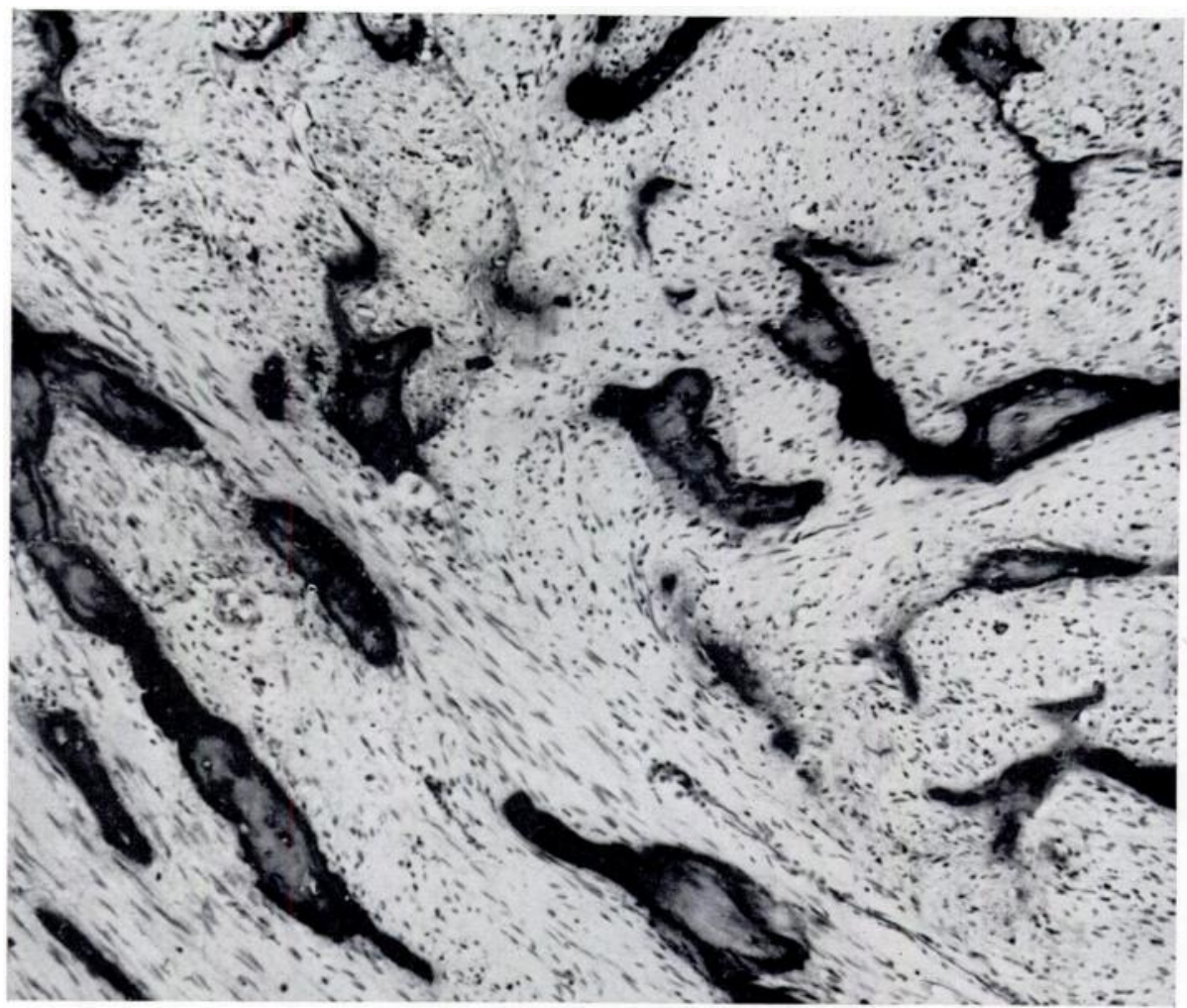

FIG. 6

The typical appearance of the tumour.

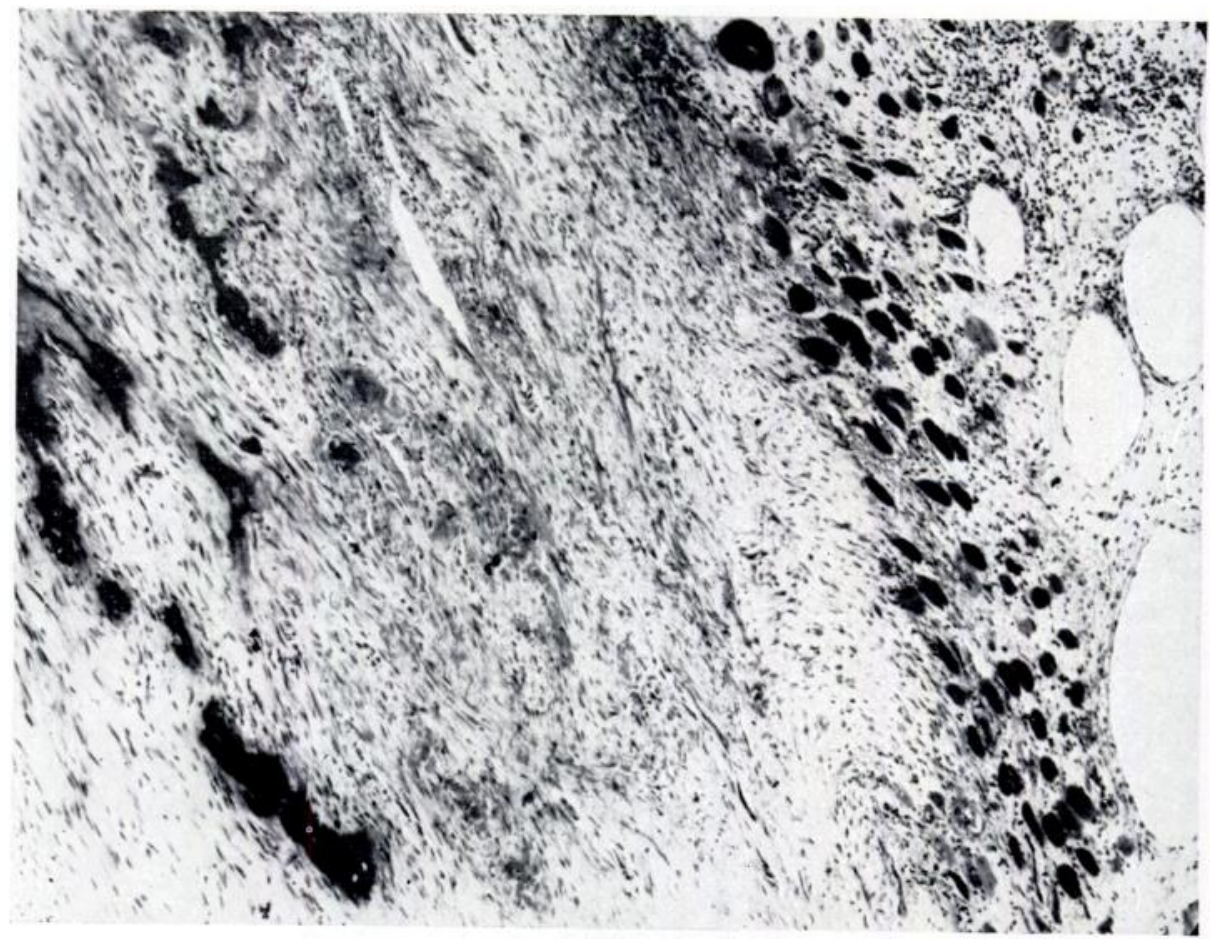

FIG. 7

Part of the proximal end of the tumour, showing infiltration of muscle and fat. 
but destroyed below this, yet without much invasion of cancellous bone. In the lateral condyle both anteriorly and laterally the cortex had been invaded.

Microscopically, some variation in histological appearances was seen in different areas. At the proximal end, cells were small and spindle-shaped with oval nuclei of very uniform appearance. Collagen fibres were dense and numerous, and there were many small irregular pieces of rather coarse new bone. Vascular spaces were small and few (Fig. 6). In adjoining areas cells were elongated and of fibroblastic type, more loosely arranged and with less collagen and bone. In still other parts the cell pattern was of herring-bone type, or gave a watered silk appearance, and here bone formation was almost absent. Only one or two very small pieces

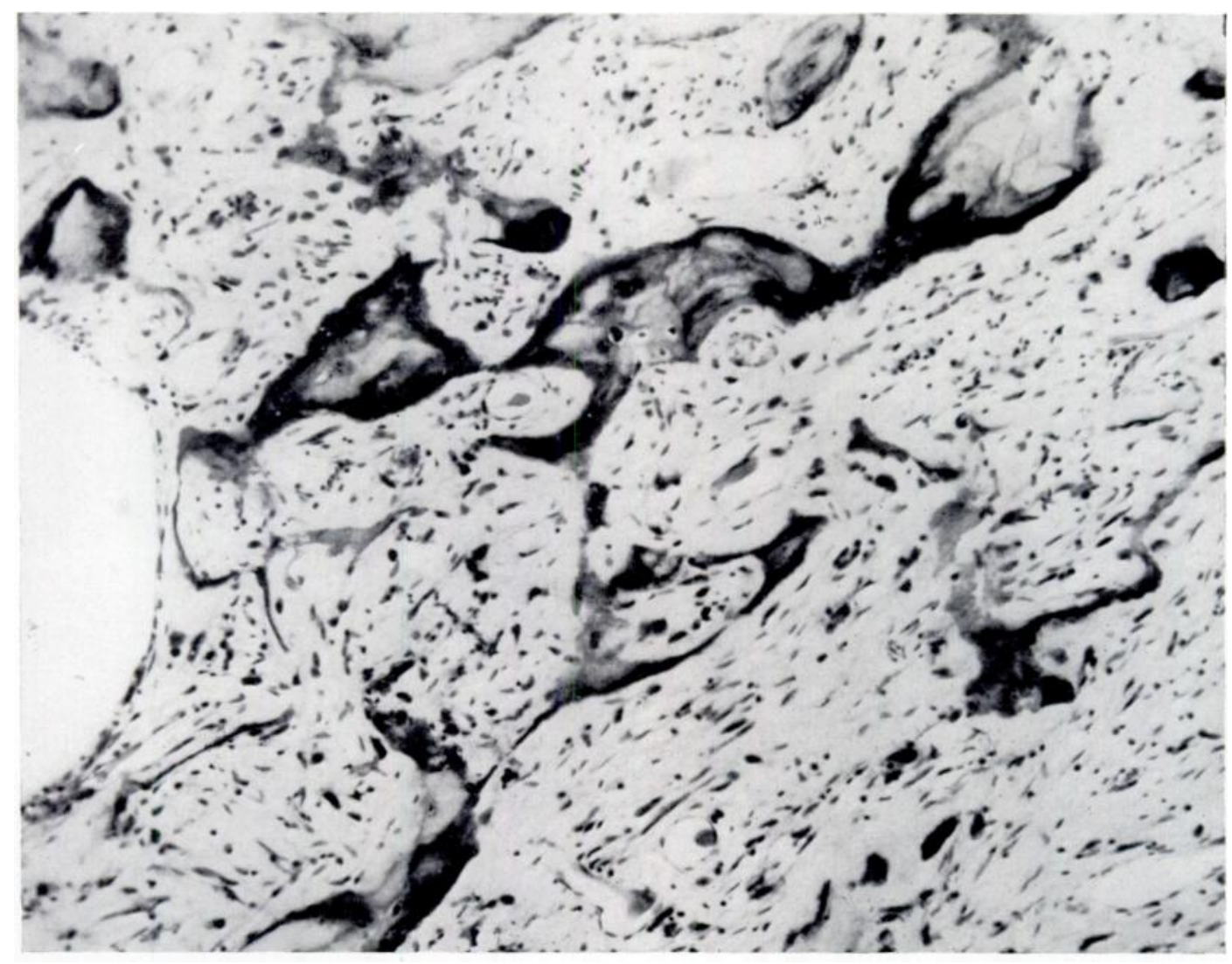

FiG. 8

Section is from the area with bone invasion. Numerous large cells with hyperchromatic nuclei of irregular shape are lying in disorderly fashion. Part of a large vascular space is present.

of cartilage were seen in the entire specimen, but bone formation was in general abundant. At the periphery there was direct infiltration of muscle (Fig. 7) and fat. Although benign in appearance in its greater part, the tumour was of different histological character in the areas where there was destruction of cortex and invasion of bone. Here (Fig. 8) the cells were larger and of variable shape, with scattered bizarre hyperchromatic single or multinucleated cells of malignant appearance. The stroma was loose and myxomatous, and bone formation was much less orderly. Very large vascular channels were seen, and appeared as endothelial-lined spaces without organised walls; apart from their size and frequency they differed in no way from the vascular spaces elsewhere in the growth.

Sections of the "bow-tie" artery, followed serially (at every $100 \mu$ ), showed localised thickening of the media, projecting into the lumen. The media appeared loose and oedematous

VOL. 40 B, NO. 4, NOVEMBER 1958 
or myxomatous with a slightly basophilic tinge to the matrix. The internal elastic lamina could be followed, at least in part, around the periphery of the invaginating mass. No inflammatory reaction or tumour invasion was seen (Fig. 9).

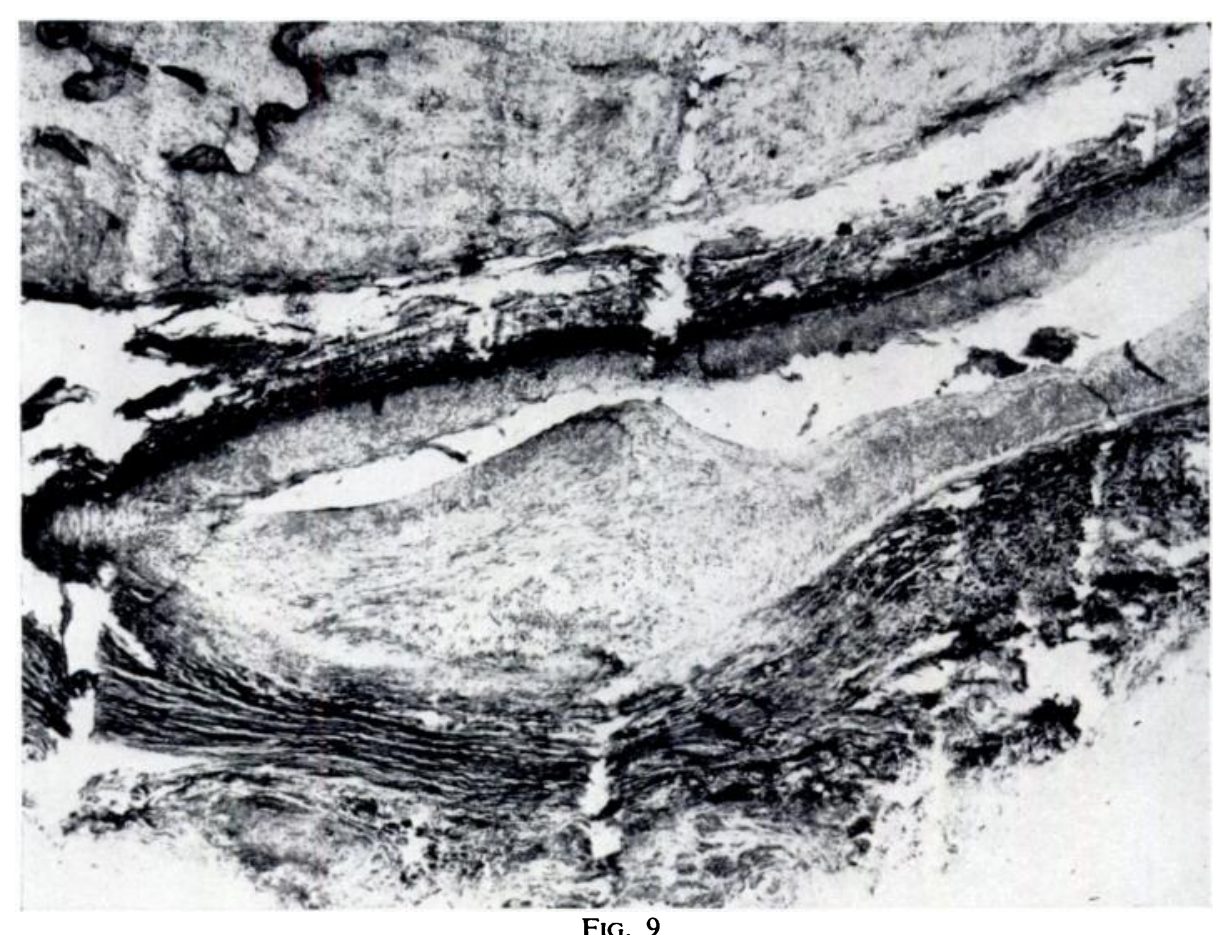

Low-power view of the " bow-tie" artery which shows the thickening of the media responsible for the constriction of the lumen.

\section{DISCUSSION}

The abnormal arteries demonstrated by the arteriograph have not before been noted in the literature. Their histological counterpart appeared as focal thickenings within the media, but they are not considered in any way specific.

Inclan (1942) and Mucchi and Columella (1951) considered that the demonstration of filled veins in the arterial phase of injection indicated malignancy. Dos Santos (1950) regarded the degree of malignancy as directly proportional to the number of arteriovenous shunts. In this case the finding of three shunts could have enabled a correct prediction of a tumour of low malignancy.

An attempt was made to see if histological appearances of malignancy were to be found in those areas where the large shunt veins were seen emerging. No such correlation could be made, but in the areas examined very large veins could be seen running in benign tumour tissue. Presumably the shunts occur at some distance from the points where confluent veins attain a size capable of radiographic demonstration.

There did appear to be good correlation between histological differentiation and the number and size of vascular channels, which in benign areas were small and few, but in malignant areas were large and numerous. But as vessels almost equally large have been seen in myositis ossificans, this finding must be regarded as non-specific. No direct arteriovenous communications were identified.

Apart from those areas with invasion of bone the appearances most closely resembled those of ossifying fibroma, a term, indeed, which was used by Copeland (1951) as a designation for the tumour. No evidence can be brought to show whether or not myositis ossificans was 
an antecedent lesion in this case and there seems to be no necessity to infer its participation. Certainly the histological appearances at the time of amputation were those of a neoplasm infiltrating muscle, fat and bone rather than any reparative type of fibrosis. The fact that only a small area with the histological criteria of malignancy could be found in a large tumour of two years' duration suggests that malignancy was of relatively recent development. It must be conceded, however, that the histological evidence of malignancy seen here is not by itself sufficient to infer clinical behaviour with accuracy. Rather, accumulated clinical experience with this type of tumour must be used to indicate what significance attaches to the microscopic picture. The degree of malignancy propounded by Dwinnell et al. (loc. cit.) is not supported by the clinical experience of Lichtenstein (loc. cit.) and for an accurate settling of these differences further experience must be awaited. In the present case, the biopsy material was regarded as benign, and amputation was done because of the radiological findings and because local removal was impractical.

The radiological diagnosis of malignancy necessitates the assumption that vascular shunts are a reliable criterion that may be applied to any growth. However, this is a relatively new approach and its degree of accuracy is not yet known. Within our own experience it would at least appear to have some limitation and require careful evaluation. Other reports of arteriography in this tumour are not available. In the most recent publications by Stevens, Pugh and Dahlin (1957) and that by Sammons, Sarkisian and Krepela (1958) no arteriographs are included.

\section{SUMMARY}

1. A case of parosteal osteoma with histologically low-grade sarcomatous areas is described.

2. Arteriography revealed abnormal arteries, the histological appearances of which are described.

3. Vascular shunts indicative of low-grade malignancy were also seen.

4. Reasons are given for accepting the view that this lesion is a tumour, originally benign, but liable to the development of low-grade malignancy.

We wish to thank Mr H. W. Wright and Dr Woodhouse Price for the histological slide of the biopsy done in London and for permission to use Dr Price's report. We also thank $\mathrm{Dr} \mathrm{H}$. A. Sissons and $\mathrm{Mr}$ G. M. Goodson for their constructive criticism of this paper and $\mathrm{Dr} \mathrm{A}$. C. Begg, who confirmed our radiological impression that this was a malignant bone tumour. Mr R. B. Sparke made reproductions of the radiographs and was responsible for the photomicrographs. Mr Philip Wrightson was responsible for the arteriograph and for the initial care of this patient.

\section{REFERENCES}

BegG, A. C. (1955): Personal communication.

Copeland, M. M. (1951): Benign Tumors of Bone. Surgery, Gynecology and Obstetrics, 90, 697.

Dwinnell, L. A., Dahlin, D. C., and Ghormley, R. K. (1954): Parosteal (Juxtacortical) Osteogenic Sarcoma. Journal of Bone and Joint Surgery, 36-A, 732.

Fine, G., and Stout, A. P. (1956): Osteogenic Sarcoma of the Extraskeletal Soft Tissues. Cancer, 9, 1,027. Geschickter, C. F., and Copeland, M.M. (1949): Tumors of Bone. Third edition. Philadelphia, London, Montreal: J. B. Lippincott Company.

Geschickter, C. F., and Copeland, M. M. (1951): Parosteal Osteoma of Bone: A New Entity. Annals of Surgery, 133, 790.

INCLAN, A. (1942): The Possibilities of the Roentgenographic Study of the Arterial Circulation in the Early Diagnosis of Bone Malignancy. Journal of Bone and Joint Surgery, 24, 259.

JAFFe, H. L., and Selin, G. (1951): Tumors of Bones and Joints. Bulletin of the New York Academy of Medicine, 27, 165.

Lichtenstein, L. (1955): Tumors of Periosteal Origin. Cancer, 8, 1,060.

Mucchi, L., and Columella, F. (1951): Arteriography in Diseases of Bone. Journal of the Faculty of Radiologists, 3, 135.

Sammons, B. P., Sarkisian, S. S., and Krepela, M. C. (1958): Juxta-cortical osteogenic sarcoma. American Journal of Roentgenology, 79, 592.

Dos Santos, R. (1950): Arteriography in Bone Tumours. Journal of Bone and Joint Surgery, 32-B, 17.

Stevens, G. M., Pugh, D. G., and Dahlin, D. C. (1957): Roentgenographic Recognition and Differentiation of Parosteal Osteogenic Sarcoma. American Journal of Roentgenology, 78, 1. 